Supporting Information

\title{
Ab Initio-Based All-Mode Two-Dimensional Infrared Spectroscopy of a Sugar Molecule
}

\author{
Jianping Wang* \\ Beijing National Laboratory for Molecular Sciences, State Key Laboratory of Molecular \\ Reaction Dynamics, Institute of Chemistry, Chinese Academy of Sciences, Beijing 100080, \\ People's Republic of China \\ jwang@iccas.ac.cn
}

\section{Ab initio computations:}

The geometry of dGA in gas phase was first fully optimized. The vibrational transition dipole moment of each mode was computed from a Hessian calculation and the corresponding harmonic normal mode frequencies were computed. The anharmonic frequencies were separately computed using a second-order vibrational perturbative approach that is available

in Gaussian03. ${ }^{1}$ All the ab initio density functional theory calculations were carried out at the level of B3LYP/6-311++G** using Gaussian03.

The atomic coordinates of the fully optimized ab initio structure of dGA in gas phase is given in Table S1. The harmonic and anharmonic frequencies and their transition dipole components of the 18 normal modes are given in Table S2. The computed anharmonicities are given in Table S3.

\section{All-mode 1D IR and 2D IR spectral computations:}

A linear response function and its Fourier transformation were used to calculate the allmode 1D IR spectra: 


$$
R_{1 D I R}=\sum_{m=1}^{3 N-6}\left|\mu_{m}\right|^{2} e^{-i \omega_{m} t-t / 2 T_{1}^{m}-g_{m}(t)}
$$

where $\omega_{m}$ is the (anharmonic) fundamental transition frequency and $T_{1}^{m}$ is the life time of the $m$ th normal mode respectively; $g_{m}(t)$ is the line shape function, having the form of $g_{m}(t)=\gamma_{m} t+\frac{1}{2} \sigma_{m}^{2} t^{2}$ when Bloch dynamics is assumed; $\gamma_{m}$ (hwhm of Lorentzian function) and $\sigma_{m}$ (variance of Gaussian function) are homogeneous and inhomogeneous broadening parameters.

Third-order nonlinear response functions were used to calculate the all-mode 2D IR spectra. A set of time-domain formulae have been derived to calculate correlated 2D IR spectra of vibrators of the same type by Hochstrasser and coworkers. ${ }^{2}$ To compute the rephasing and non-rephasing 2D IR spectra of the complete $3 N-6$ normal modes of different types, we start from four-wave mixing experiment. In the four-wave mixing 2D IR experiment, three incident infrared ultrashort laser pulses with wave vectors $\hat{k}_{1}, \hat{k}_{2}$, and $\hat{k}_{3}$, delayed by $\tau$ (coherence time) and $T$ (population time or waiting time), excite the vibrational level $|0\rangle \rightarrow|m\rangle,|m\rangle \rightarrow|m+m\rangle$ and $|m\rangle \rightarrow|m+n\rangle$ for $m=1,2, \ldots, 3 N-6$. The generated field $E(\tau, T, t)$ has a wave vector $\hat{k}_{s}$, satisfying a phase-matching condition: $\hat{k}_{s}=-\hat{k}_{1}+\hat{k}_{2}+\hat{k}_{3}$. Here $t$ is the 2D IR signal detection time. The third-order response $R(\tau, T, t)$ is proportional to the real part of the generated field $E(\tau, T, t)$. The Feynman diagrams of Liouville-space pathways of the rephasing and non-rephasing 2D IR signals at $T=0$ are given in Figure $1 S^{3}{ }^{3}$ The diagrams representing the 2D IR signals with overlapping laser pulses are not considered in 
Figure 1S, because their contributions to signal at zero time are negligible when laser pulses are narrow in the case of a broadband 2D IR experiment.

The total third-order response is the sum of the response function of each individual path. Signals $\mathrm{R}_{1}$ to $\mathrm{R}_{6}$ for the rephasing diagrams can be summed as

$$
\sum_{i=1}^{6} R_{i}(\tau, t)=i \sum_{m \neq n}^{3 N-6} e^{i \omega_{m} \tau}\left\{\begin{array}{l}
\left\langle a_{m} b_{m} c_{m} d_{m}\right\rangle\left[2 L(0 m|00| m 0)-L(0 m|m m| m+m, m) e^{i \Delta_{m} t}\right] e^{-i \omega_{m} t}+ \\
\left\langle a_{m} b_{n} c_{m} d_{n}\right\rangle\left[L(0 m|n m| n 0)-L(0 m|n m| n+m, m) e^{i \Delta_{m n} t}\right] e^{-i \omega_{n} t}+ \\
\left\langle a_{m} b_{m} c_{n} d_{n}\right\rangle\left[L(0 m|00| n 0)-L(0 m|m m| n+m, m) e^{i \Delta_{m n} t}\right] e^{-i \omega_{n} t}
\end{array}\right\},
$$

whereas signals $\mathrm{R}_{7}$ to $\mathrm{R}_{9}$ for the non-rephasing diagrams can be summed as

$$
\sum_{i=7}^{9} R_{i}(\tau, t)=i \sum_{m \neq n}^{3 N-6} e^{-i \omega_{m} \tau}\left\{\begin{array}{l}
\left\langle a_{m} b_{m} c_{m} d_{m}\right\rangle\left[2 L(m 0|00| m 0)-L(m 0|m m| m+m, m) e^{i \Delta_{m} t}\right] e^{-i \omega_{m} t}+ \\
\left\langle a_{m} b_{n} c_{n} d_{m}\right\rangle\left[L(m 0|n m| m 0)-L(m 0|n m| n+m, n) e^{i \Delta_{m n} t}\right] e^{-i \omega_{m} t}+ \\
\left\langle a_{m} b_{m} c_{n} d_{n}\right\rangle\left[L(m 0|00| n 0)-L(m 0|m m| n+m, m) e^{i \Delta_{m n} t}\right] e^{-i \omega_{n} t}
\end{array}\right\},
$$

For a system with 3N-6 normal modes, there are 3N-6 one-quantum states and $3(N / 2-1)(3 N-5)$ two-quantum states. In these equations, $\Delta_{m}$ and $\Delta_{m n}$ are the diagonal and offdiagonal anharmonicities respectively. Forbidden transitions such as $|m\rangle \rightarrow|n+p\rangle$ where (where $n, p \neq m$ ) are not considered when constructing 2D IR spectra in the basis of anharmonicities, which is significantly different from the case using vibrational exciton model. ${ }^{3-4}$ In these equations, the vibrational dynamics are included in the Liouville factor $L(m n|p q| r s)$. Vibrational relaxation among one-quantum or two-quantum states and rotational dephasing processes are ignored. The factor also contains the product of the transition dipoles involved in the Liouville path, giving the 2D IR signal strength. Using Bloch dynamics, the Liouville factor $L(m n|p q| r s)$ in Eqs. S2 to S3 can be written as 


$$
\begin{aligned}
& L\left(\left\{\begin{array}{l}
0 m \\
m 0
\end{array}\right\}|00| m 0\right)=\left|\mu_{m}\right|^{4} \exp \left[-\left(\frac{1}{2 T_{1}^{m}}+\gamma_{m}\right)(\tau+t)-\frac{1}{2} \sigma_{m}^{2}(\tau \mp t)^{2}\right] \\
& L\left(\left\{\begin{array}{l}
0 m \\
m 0
\end{array}\right\}|m m| m+m, m\right)=2 L\left(\left\{\begin{array}{l}
0 m \\
m 0
\end{array}\right\}|00| m 0\right) \exp \left[-\frac{1}{2 T_{1}^{m+m}} t\right] \\
& L\left(\left\{\begin{array}{l}
0 m \\
m 0
\end{array}\right\}|00| n 0\right)=\left|\mu_{m}\right|^{2}\left|\mu_{n}\right|^{2} \exp \left[-\left(\frac{1}{2 T_{1}^{m}}+\gamma_{m}\right) \tau-\left(\frac{1}{2 T_{1}^{n}}+\gamma_{n}\right) t-\frac{1}{2} \sigma_{m}^{2} \tau^{2}-\frac{1}{2} \sigma_{n}^{2} t^{2} \pm f_{m n} \sigma_{m} \sigma_{n} \tau t\right]
\end{aligned}
$$

$$
\begin{aligned}
& L\left(\left\{\begin{array}{l}
0 m \\
m 0
\end{array}\right\}\left|\left\{\begin{array}{l}
m m \\
n m
\end{array}\right\}\right| n+m, m\right)=L\left(\left\{\begin{array}{l}
0 m \\
m 0
\end{array}\right\}|00| n 0\right) \exp \left[-\left(\frac{1}{2 T_{1}^{m+n}}+\frac{1}{2 T_{1}^{m}}-\frac{1}{2 T_{1}^{n}}\right) t\right] \\
& L(m 0|n m| m 0)=\left|\mu_{m}\right|^{2}\left|\mu_{n}\right|^{2} \exp \left[-\left(\frac{1}{2 T_{1}^{m}}+\gamma_{m}\right)(\tau+t)-\frac{1}{2} \sigma_{m}^{2}(\tau+t)^{2}\right] \\
& L(0 m|n m| n 0)=L(0 m|00| n 0)
\end{aligned}
$$

Here, we assume $\frac{1}{T_{1}^{m+m}}=\frac{2}{T_{1}^{m}}$ and $\frac{1}{T_{1}^{m+n}}=\frac{1}{T_{1}^{m}}+\frac{1}{T_{1}^{n}}$ using a harmonic oscillator

approximation. Polarization factor $\left\langle a_{\alpha} b_{\beta} c_{\chi} d_{\delta}\right\rangle$ determines the final peak intensity, depending on laboratory polarization of four laser pulses $a, b, c$, and $d$, and the angles $\alpha, \beta, \chi$, and $\delta$ of the transition dipoles in molecular frame. To calculate polarized 2D IR spectra, the polarization factor for a 2D IR signal under the $<$ zzzz $>$ polarization is $1 / 5$ for the diagonal signals and $\left(4 P_{2}+5\right) / 45$ for the off-diagonal signals, ${ }^{5}$ where $P_{2}=\left(3 \cos \theta_{m n}{ }^{2}-1\right) / 2$ is second Legendre polynomial; and $\theta_{m n}$ is the angle between $\vec{\mu}_{m}$ and $\vec{\mu}_{n}$. The <zzzz> polarization means four laser pulses (three incident plus a local oscillator for heterodyned signal detection) having their electric fields all in vertical direction. The polarization factor for a 2D IR signal under the $<z x x z>$ polarization is $1 / 15$ for the diagonal signals and $P_{2} / 15$ for the off-diagonal 
signals. Here $<z x x z>$ indicates that the second and third pulses have their electric fields all in horizontal direction.

The total (absorptive) correlation 2D IR signal at $T=0$ is given by a double Fourier transform of the total response function:

$$
S\left(\omega_{\tau}, \omega_{t}\right)=\int_{0}^{+\infty} e^{i \omega_{\tau}} d \tau \int_{0}^{+\infty} e^{i \omega_{t}} d t \sum_{i=1}^{9} R_{i}(\tau, t)
$$

The transition dipole moments for 18 modes obtained from ab initio Hessian computations (Table S2) were used in above equations. We also assume $\mu_{m+m}^{2}=2 \mu_{m}^{2}$ in formulating the response functions. We use the ab initio-computed anharmonicities ( $\Delta_{m}$ and $\Delta_{m n}$ ) given in Table S3 in the corresponding third-order nonlinear response functions that involve the overtone and combination state transitions (Eqs. S2 to S3).

The correlation of the inhomogeneous $0 \rightarrow 1$ transition frequency distributions was introduced by the statistical correlation coefficient $f_{m n}$ in the response functions (Eq. S6); pairwise correlation coefficients $f_{m n}$, defined as $\left\langle\delta \omega_{m} \delta \omega_{n}\right\rangle / \sigma_{m} \sigma_{n}$, can be adjusted freely for the entire 18 modes; they were set to +1 except that for the $\mathrm{C}=\mathrm{O}$ stretching mode (mode 14 ) and the C-D stretching mode (mode 15 ), for that we set $f_{14,15}=-0.35$ in our spectral computation. We found that by using this value our simulation can reproduce the spectral feature of the $\mathrm{C}=\mathrm{O} / \mathrm{C}-\mathrm{D}$ cross peak in formamide- $\mathrm{d}_{3}$ well. ${ }^{6}$ We set $\gamma_{m}=7 \mathrm{~cm}^{-1}$ and $\sigma_{m}=25 \mathrm{~cm}^{-1}$ as the homogeneous and inhomogeneous broadenings for each mode under the Bloch dynamics assumption. The vibration relaxation lifetime $\left(T_{1}^{m}\right)$ in the response functions for each mode can also be adjusted freely, which was all set to 1000 fs in the present study. These parameters could be given more reasonable values whenever possible. 
The computed absorptive 2D IR spectrum with $<z x x z>$ polarization is shown in Figure S2, the enlarged region for the $\mathrm{C}=\mathrm{O} / \mathrm{C}-\mathrm{D}$ stretching modes is given in Figure S3a and that for the $\mathrm{C}-\mathrm{D} / \mathrm{O}-\mathrm{H}$ in-plane-bending modes is given in Figure S3b, respectively. 
Table S1. Optimized atomic coordinates of dGA in gas phase

\begin{tabular}{|c|c|c|c|c|}
\hline & atom & $\mathrm{x}$ & $\mathrm{y}$ & $\mathrm{z}$ \\
\hline 1 & $\mathrm{O}$ & -0.772 & 0.411 & -1.204 \\
\hline 2 & $\mathrm{C}$ & -0.776 & 0.571 & -0.006 \\
\hline 3 & $\mathrm{C}$ & 0.252 & -0.068 & 0.891 \\
\hline 4 & $\mathrm{O}$ & 1.181 & -0.846 & 0.186 \\
\hline 5 & $\mathrm{D}$ & -1.540 & 1.199 & 0.492 \\
\hline 6 & $\mathrm{H}$ & -0.285 & -0.677 & 1.636 \\
\hline 7 & $\mathrm{H}$ & 0.755 & 0.735 & 1.451 \\
\hline 8 & $\mathrm{H}$ & 0.941 & -0.792 & -0.751 \\
\hline
\end{tabular}

Table S2. Calculated fundamental harmonic $\left(\omega_{h}\right)$ and anharmonic $(\omega)$ frequencies (in $\mathrm{cm}^{-1}$ ); calculated transition dipole moment $\mu$ (in $\mathrm{D}^{-1} \mathrm{amu}^{-1 / 2}$ ) and its component of the $18 \mathrm{normal}$ modes in gas-phase dGA

\begin{tabular}{|c|c|c|c|c|c|c|}
\hline \multirow{2}{*}{$\begin{array}{c}\text { Normal } \\
\text { mode }\end{array}$} & \multirow{2}{*}{$\omega_{h}$} & $\omega$ & \multicolumn{4}{|c|}{$\mu$} \\
\cline { 4 - 7 } & & & $|\mu|$ & $\mu_{\mathrm{x}}$ & $\mu_{\mathrm{y}}$ & $\mu_{\mathrm{z}}$ \\
\hline 1 & 183.596 & 187.259 & 0.491893 & -0.00030 & 0.000242 & 0.491893 \\
\hline 2 & 278.103 & 268.228 & 0.739003 & -0.53082 & -0.51415 & 0.000304 \\
\hline 3 & 368.993 & 298.455 & 1.558821 & 0.00040 & -0.00038 & -1.55882 \\
\hline 4 & 630.864 & 621.676 & 0.208979 & 0.00000 & 0.00010 & 0.208979 \\
\hline 5 & 743.176 & 728.681 & 0.622890 & 0.621062 & -0.04769 & 0.00000 \\
\hline 6 & 798.389 & 771.293 & 0.669033 & 0.533884 & 0.403204 & 0.000224 \\
\hline 7 & 1029.369 & 1007.537 & 0.004730 & $-6.8 \mathrm{E}-05$ & -0.00022 & -0.00472 \\
\hline 8 & 1093.121 & 1081.074 & 1.332042 & 0.948535 & 0.93521 & 0.000119 \\
\hline 9 & 1127.457 & 1101.868 & 1.081103 & 0.431787 & 0.991133 & -0.00022 \\
\hline 10 & 1240.4 & 1212.522 & 0.245042 & -0.00032 & -0.00017 & -0.24504 \\
\hline 11 & 1281.88 & 1251.876 & 0.855986 & -0.81442 & -0.26351 & 0.00000 \\
\hline 12 & 1420.362 & 1377.722 & 1.221896 & -0.27449 & -1.19067 & 0.000149 \\
\hline 13 & 1468.931 & 1431.147 & 0.679845 & -0.67981 & -0.00694 & 0.000319 \\
\hline 14 & 1774.894 & 1744.594 & 1.978229 & 1.344754 & -1.45087 & -0.00052 \\
\hline 15 & 2167.162 & 2028.947 & 1.290757 & -0.49846 & -1.19063 & 0.001077 \\
\hline 16 & 2976.354 & 2865.743 & 0.944677 & 0.261265 & 0.90783 & -0.00027 \\
\hline 17 & 2992.072 & 2828.866 & 0.669029 & 0.000742 & 0.000191 & 0.669029 \\
\hline 18 & 3724.431 & 3531.642 & 1.246813 & 0.987840 & -0.76073 & 0.00000 \\
\hline
\end{tabular}


Table S3. Calculated diagonal and off-diagonal anharmonicities $\Delta_{m}$ and $\Delta_{m n}\left(\right.$ in $\mathrm{cm}^{-1}$ ) of the 18 normal modes in dGA

\begin{tabular}{|c|c|c|c|c|c|c|c|c|c|c|c|c|c|c|c|c|c|c|}
\hline $\mathrm{m} / \mathrm{n}$ & 1 & 2 & 3 & 4 & 5 & 6 & 7 & 8 & 9 & 10 & 11 & 12 & 13 & 14 & 15 & 16 & 17 & 18 \\
\hline 1 & -4.794 & 5.826 & 8.365 & -15.222 & 6.311 & 11.093 & 2.097 & 0.269 & 1.412 & -7.748 & -4.497 & -9.722 & -3.512 & -1.042 & 3.533 & -2.532 & -2.601 & -11.201 \\
\hline 2 & & -1.047 & 14.587 & 0.131 & 1.571 & 9.146 & 0.391 & -6.423 & 1.959 & -2.009 & -1.067 & -13.266 & 0.564 & -0.25 & 2.343 & 0.069 & 0.131 & -2.404 \\
\hline 3 & & & 74.797 & 1.463 & 4.548 & 1.136 & 8.648 & -0.318 & -0.816 & -5.354 & -6.575 & -12.754 & 1.901 & -4.591 & 10.2 & -1.278 & -2.904 & -34.622 \\
\hline 4 & & & & -0.835 & 5.933 & 10.376 & 4.589 & -0.404 & -0.385 & -1.285 & -0.242 & -9.936 & 2.463 & 1.155 & 5.354 & 3.323 & 2.601 & -0.412 \\
\hline 5 & & & & & 1.04 & 1.995 & 0.567 & 0.717 & 3.235 & 0.723 & 1.782 & -8.538 & 1.168 & 4.276 & 2.675 & -0.572 & -1.001 & -1.197 \\
\hline 6 & & & & & & 3.233 & 2.27 & -4.568 & 1.641 & 0.988 & 3.125 & -5.704 & 0.301 & 1.616 & 4.75 & -0.226 & -0.806 & 0.029 \\
\hline 7 & & & & & & & 4.8 & 1.153 & 0.569 & -0.883 & 0.373 & -17.587 & 7.832 & 2.983 & 0.049 & 6.068 & 4.287 & 0.107 \\
\hline 8 & & & & & & & & -34.754 & -0.18 & 1.8 & 2.709 & -8.228 & 1.107 & 5.962 & 91.711 & -0.438 & -1.029 & -0.784 \\
\hline 9 & & & & & & & & & 8.178 & 7.265 & 6.893 & -8.491 & 1.189 & 2.596 & 7.404 & 0.153 & -0.389 & 0.205 \\
\hline 10 & & & & & & & & & & 4.836 & 9.084 & -4.133 & 6.594 & 1.136 & -0.002 & 10.169 & 4.893 & 3.415 \\
\hline 11 & & & & & & & & & & & 7.997 & 6.552 & 3.511 & 0.415 & 1.051 & -5.387 & 1.21 & 14.532 \\
\hline 12 & & & & & & & & & & & & -2.951 & -8.171 & -10.478 & -9.229 & -27.692 & -6.533 & -4.966 \\
\hline 13 & & & & & & & & & & & & & 40.441 & 1.079 & 0.404 & -50.357 & 17.221 & 0.835 \\
\hline 14 & & & & & & & & & & & & & & 19.842 & -0.967 & 1.617 & 2.222 & 2.635 \\
\hline 15 & & & & & & & & & & & & & & & 77.662 & -1.933 & -2.084 & -5.047 \\
\hline 16 & & & & & & & & & & & & & & & & 63.295 & 150.383 & 2.696 \\
\hline 17 & & & & & & & & & & & & & & & & & 73.881 & 2.504 \\
\hline 18 & & & & & & & & & & & & & & & & & & 204.268 \\
\hline
\end{tabular}




\section{Rephasing}
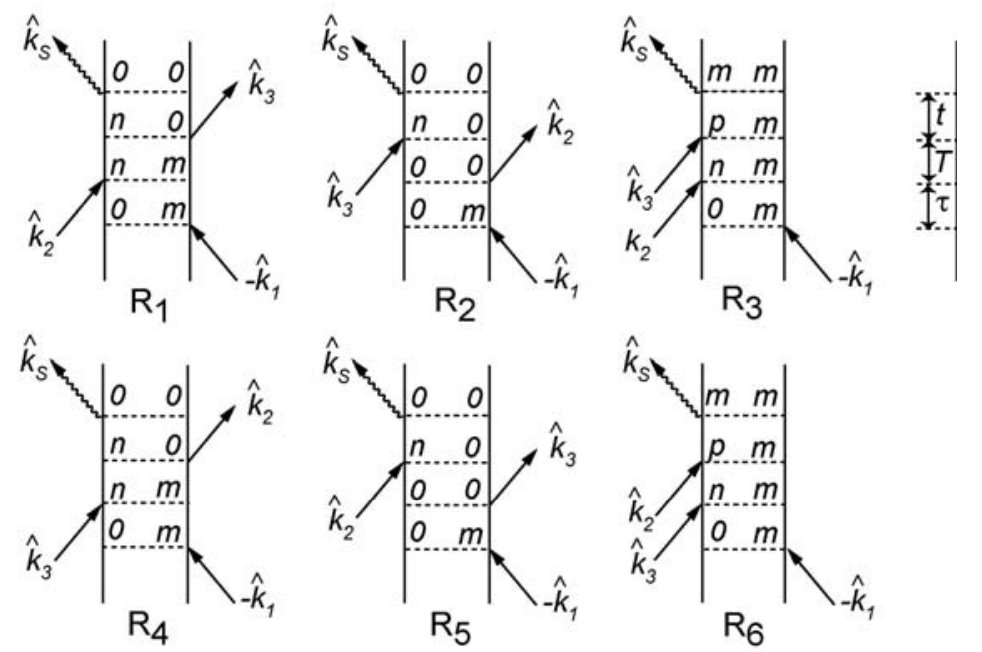

Nonrephasing
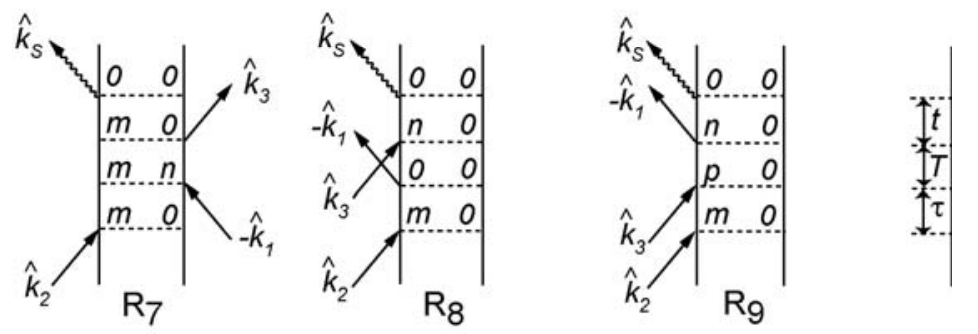

Figure S1. Feynman diagrams for Liouville-space pathways of the rephasing and nonrephasing 2D IR signals at $T=0$. The first excited states are denoted as $m$ and $n$; the overtone and combination states are denoted as $p=m+m$ and $p=m+n$. Three incident laser pulses and the signal field are shown to have wave vectors as $\hat{k}_{1}, \hat{k}_{2}, \hat{k}_{3}$, and $\hat{k}_{s}$. 


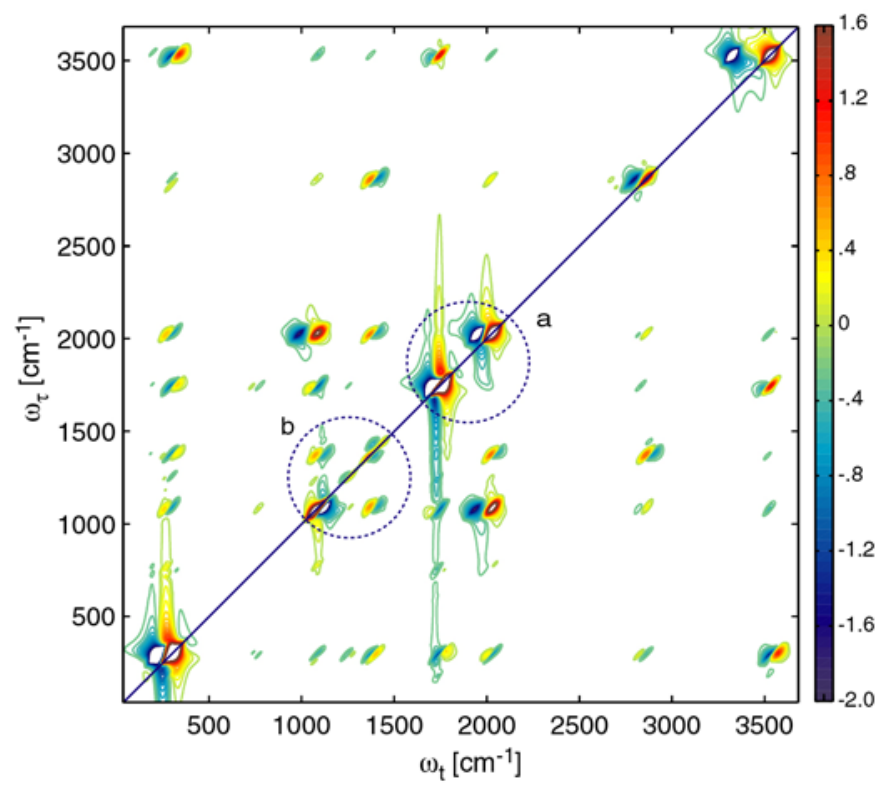

Figure S2. All-mode absorptive 2D IR spectrum of dGA with $<$ zxxz $>$ polarization. Circles show two selected small regions: (a) $\mathrm{C}=\mathrm{O}$ and $\mathrm{C}-\mathrm{D}$ stretching modes; (b) $\mathrm{C}-\mathrm{D}$ and $\mathrm{O}-\mathrm{H}$ in-plane-bending modes.

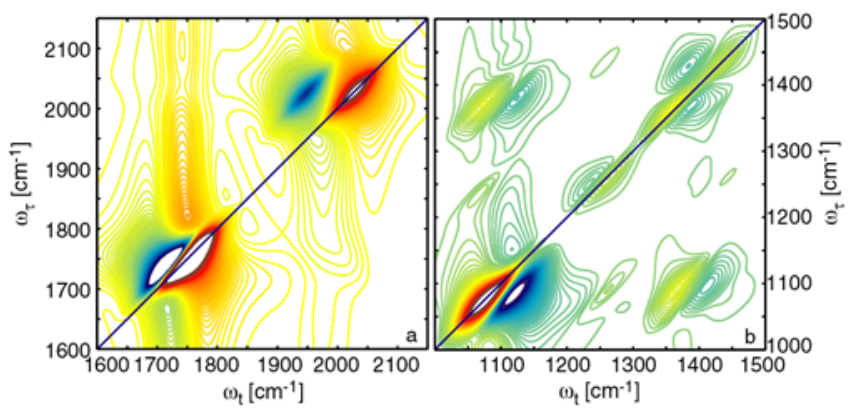

Figure S3. Enlarged regions ( $\mathrm{a}$ and $\mathrm{b}$ ) marked in Figure S2 to show the dynamics and correlated frequency distributions between the selected modes. 


\section{References}

(1) Frisch, M. J.; Trucks, G. W.; Schlegel, H. B.; Scuseria, G. E.; Robb, M. A.;

Cheeseman, J. R.; Vreven, J. T.; K. N. Kudin; J. C. Burant; J. M. Millam; S. S. Iyengar; J.

Tomasi; V. Barone; B. Mennucci; M. Cossi; G. Scalmani; N. Rega; G. A. Petersson; H.

Nakatsuji; M. Hada; M. Ehara; K. Toyota; R. Fukuda; J. Hasegawa; M. Ishida; T.

Nakajima; Y. Honda; O. Kitao; H. Nakai; M. Klene; X. Li; J. E. Knox; H. P. Hratchian; J.

B. Cross; C. Adamo; J. Jaramillo; R. Gomperts; R. E. Stratmann; O. Yazyev; A. J. Austin;

R. Cammi; C. Pomelli; J. W. Ochterski; P. Y. Ayala; K. Morokuma; G. A. Voth; P.

Salvador; J. J. Dannenberg; V. G. Zakrzewski; S. Dapprich; A. D. Daniels; M. C. Strain;

O. Farkas; D. K. Malick; A. D. Rabuck; K. Raghavachari; J. B. Foresman; J. V. Ortiz; Q.

Cui; A. G. Baboul; S. Clifford; J. Cioslowski; B. B. Stefanov; G. Liu, A.; Liashenko; P.

Piskorz; I. Komaromi; R. L. Martin; D. J. Fox; T. Keith; M. A. Al-Laham; C. Y. Peng; A. Nanayakkara; M. Challacombe; P. M. W. Gill; B. Johnson; W. Chen; M. W. Wong; C.

Gonzalez; Pople, J. A., Gaussian 03, Revision B.05.; Gaussian, Inc., Pittsburgh PA, 2003.

(2) Ge, N.-H.; Zanni, M. T.; Hochstrasser, R. M., J. Phys. Chem. A 2002, 106, 962-972.

(3) Wang, J.; Hochstrasser, R. M., Chem. Phys. 2004, 297, 195-219.

(4) Hamm, P.; Lim, M.; Hochstrasser, R. M., J. Phys. Chem. B 1998, 102, 6123-6138.

(5) Hochstrasser, R. M., Chem. Phys. 2001, 266, 273-284.

(6) Kumar, K.; Sinks, L. E.; Wang, J.; Kim, Y. S.; Hochstrasser, R. M., Chem. Phys. Lett. 2006, 432, 122-127. 